\title{
Identifiability Implies Robust Identifiability
}

\author{
Lennart Ljung, Torkel Glad and Torbjörn Andersson \\ email: ljung@isy.liu.se torkel@isy.liu.se toand@isy.liu.se \\ Department of Electrical Engineering \\ Linköping University \\ S-581 83 Linköping, Sweden
}

Keywords: identification, bounded disturbances, robustly convergent, global identifiability, robust global identifiability.

\section{Identification and robustly convergent algorithms}

There has been a recent interest in identification from a deterministic perspective, like $H_{\infty}$ - techniques (Helmicki et al., 1991), (Helmicki et al., 1992), (Gu and Khargonekar, 1992), $L_{1}$ - techniques (Mäkilä, 1991), (Chen et al., 1992) etc. One concept that has been defined in that context is robustly convergent identification algorithms (tuned or untuned), see (Helmicki et al., 1991).

The concept aims at capturing the following property: Suppose a true system $G_{0} \in \mathcal{G}$ generates exact inputoutput data

$$
Z_{0}^{N}=\left\{y_{0}(t), u(t) ; t=1, \ldots, N\right\}
$$

Here $\mathcal{G}$ is a set of systems. Suppose also that the data are subjected to errors $v(t)$ :

$$
\begin{gathered}
y(t)=y_{0}(t)+v(t) \\
Z^{N}=\{y(t), u(t) ; t=1, \ldots, N\}
\end{gathered}
$$

Let $\mathcal{A}$ be an identification algorithm, i.e a mapping from $Z^{N}$ to a space of models:

$$
\hat{G}_{N}=\mathcal{A}\left(Z^{N}\right)
$$

This algorithm is then said to be robustly convergent (Helmicki et al., 1991) if

$$
\begin{gathered}
\lim _{\delta \rightarrow 0} \lim _{N \rightarrow \infty}\left\|\hat{G}_{N}-G_{0}\right\|=0 \\
|v(t)| \leq \delta \quad \forall t
\end{gathered}
$$

for all $G_{0} \in \mathcal{G}$. The algorithm is "untuned" if it does not use knowledge of either $\mathcal{G}$ or $\delta$.

In (5) $\|\cdot\|$ denotes a suitable norm, for linear systems, it is typically chosen as the $H_{\infty}-\operatorname{norm}\left(\sup _{\omega} \mid \hat{G}_{t}\left(e^{i \omega}\right)-\right.$ $\left.G_{0}\left(e^{i \omega}\right) \mid\right)$.

Now traditional identification theory has paid substantial attraction to (5). In, e.g. (Ljung and Yuan, 1985)
(Thm 3.3), (5) is established to hold (with probability 1 ) without the limit $\delta$, provided (6) is replaced by an assumption that $\{v(t)\}$ is a stochastic process with a covariance function that decays sufficiently fast. In (Ljung and Yuan, 1985) a least squares algorithm based on a FIR model is used, and similar results are obtained for ARX - models in (Ljung and Wahlberg, 1992).

Khargonekar and Akçay have established (6) and (5) for ARX - models, see (Akçay, 1992). We shall in the next section show how this can be proved within a "classical identification framework".

Another and major part of this contribution is however that the linear regression (ARX or FIR case) also sets the pattern for a very general "robust identifiability" problem in the spirit of (6) and (5). We could formulate this as follows: Let $\mathcal{M}$ be a certain model structure, i.e. a smoothly parametrized set of models

$$
\mathcal{M}=\left\{\mathcal{M}(\theta) \mid \theta \in D_{\mathcal{M}}\right\}
$$

Let a system $\rho=\mathcal{M}\left(\theta_{0}\right)$ generate exact data $Z_{0}^{N}$ and let it be disturbance corrupted as in (1), (2) and (3). We would then say that $\mathcal{M}$ is globally identifiable at $\theta_{0}$ if there exists an input $u(t)$, such that the value $\theta_{0}$ can uniquely (globally) be recovered from the identification in $Z_{0}^{N}$. We could say that it is robustly globally identifiable if there exists an identification algorithm $\mathcal{J}$ and a $N$ such that

$$
\begin{gathered}
\hat{\theta}_{N}=\mathcal{J}\left(Z^{N}\right) \\
\lim _{\delta \rightarrow 0}\left|\hat{\theta}_{N}-\theta_{0}\right|=0
\end{gathered}
$$

More precise definitions will be given in section 3 .

A conceptually interesting result is that

Global identifiability $\Rightarrow$ Robust global identifiability

In section 4 it is described how (10) can be established.

\section{The least squares algorithm is globally convergent}

It is here proved for both the continuous- and discrete case simultaneously that the least squares algorithm is 
robustly convergent for ARX-models. Let the letter $\sigma$ Let

denote either the differential operator $p=\frac{d}{d t}$ or the shift operator $q(q y(t)=y(t+1))$. Consider the ARX-model

$$
A(\sigma) y(t)=B(\sigma) u(t)
$$

where

$$
\begin{gathered}
A(\sigma)=\sigma^{n}+a_{1} \sigma^{n-1}+\cdots+a_{n} \\
B(\sigma)=b_{1} \sigma^{n-1}+\cdots+b_{n}
\end{gathered}
$$

Let

$$
\begin{gathered}
\varphi(t)=\left[-\sigma^{n-1} y(t),-\sigma^{n-2} y(t), \ldots,-y(t),\right. \\
\left.\sigma^{n-1} u(t), \ldots, u(t)\right]^{T} \\
\theta=\left[a_{1}, a_{2}, \ldots, a_{n}, b_{1}, \ldots, b_{n}\right]^{T} .
\end{gathered}
$$

The model (11) can then be written

$$
\sigma^{n} y(t)=\theta^{T} \varphi(t)
$$

The least squares solution to (11)-(13) can be written

$$
\hat{\theta}_{N}=\underbrace{\left[\frac{1}{N} \sum_{k=1}^{N} \varphi(k) \varphi^{T}(k)\right]^{-1}}_{R_{N}^{-1}} \frac{1}{N} \sum_{k=1}^{N} \varphi(k) \sigma^{n} y(k)
$$

provided the inverse $R_{N}^{-1}$ exists. The conditions on $\{u(t)\}$ such that $R_{N}$ is uniformly positive definite are called persistence of excitation (p.e.)(Åström and Wittenmark, 1989), (Ljung, 1987).

Let

$$
\tilde{u}(t)=\frac{1}{A(\sigma)} u(t)
$$

so that

$$
y(t)=B(\sigma) \tilde{u}(t)
$$

We then have

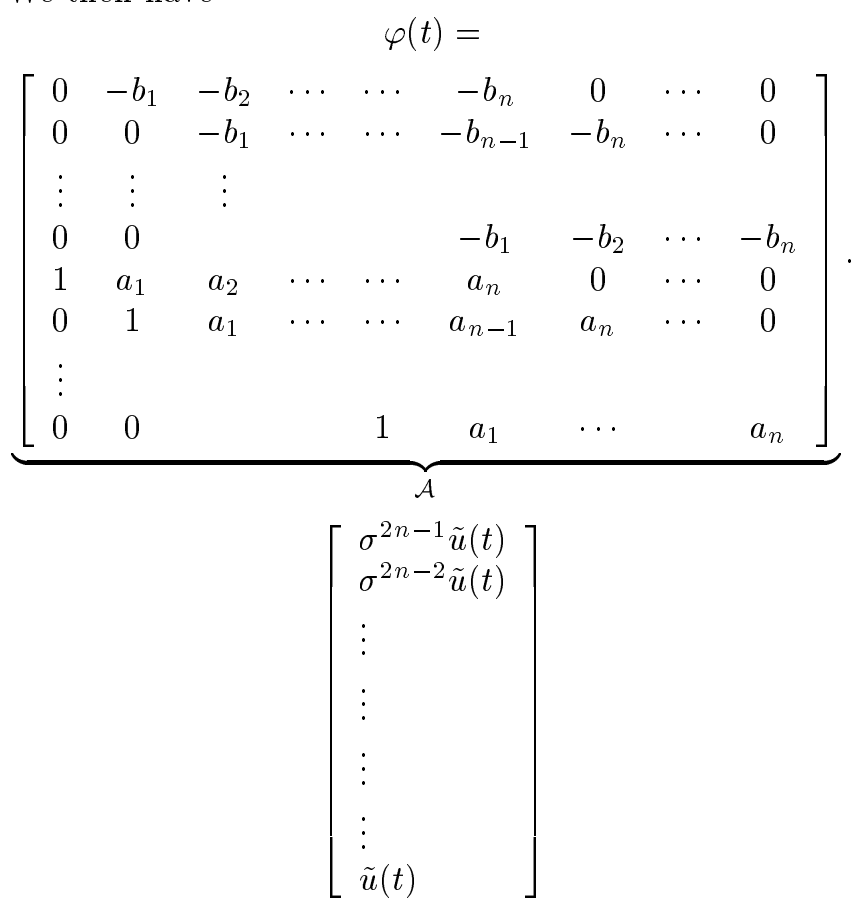

$$
\tilde{\varphi}(t)=\left[\sigma^{2 n-1} \tilde{u}(t) \ldots \tilde{u}(t)\right]^{T}
$$

so

$$
\varphi(t)=\mathcal{A} \tilde{\varphi}(t)
$$

The Sylvester matrix $\mathcal{A}$ is non-singular if $A(\sigma)$ and $B(\sigma)$ have no common factors. Hence

$$
R_{N} \geq \delta_{1} I
$$

if

$$
\tilde{R}_{N}=\frac{1}{N} \sum_{t-1}^{N} \tilde{\varphi}(t) \tilde{\varphi}^{T}(t) \geq \delta_{2} I
$$

The condition (15) is known as $\tilde{u}(t)$ is p.e. of order $2 n$ (sometimes the definition is applied to the limit as $N \rightarrow$ $\infty$, but we should here let it hold uniformly in $N \geq N_{0}$ which is the same for large enough $N_{0}$ ). In view of (14) $\tilde{u}(t)$ is p.e. of order $2 n$ if $u(t)$ is, provided $A(\sigma)$ has no zeros on the unit circle (no zeros on the imaginary axis).

There are many ways to generate sequences $\{u(t)\}$ that are p.e. as required (Åström and Wittenmark, 1989), (Ljung, 1987). Now suppose that we have a disturbance

$$
A(\sigma) y(t)=B(\sigma) u(t)+v(t)
$$

where $|v(t)|<\delta, \quad 0 \leq \delta<\infty$. in the discrete case and in addition $\left|v^{(j)}(t)\right|<c_{j} \delta \quad 0 \leq \delta, c_{j}<\infty$ for the continuous case. Let

$$
y(t)=y_{u}(t)+y_{v}(t)
$$

where

$$
\begin{gathered}
A(\sigma) y_{u}(t)=B(\sigma) u(t) \\
A(\sigma) y_{v}(t)=v(t)
\end{gathered}
$$

If $A(\sigma)$ has no roots on the unit circle (no roots on the imaginary axis) we have

$$
\left|y_{v}(t)\right| \leq \delta_{3} \cdot \max |v(t)| \leq \delta_{3} \cdot \delta
$$

Let

$$
\varphi(t)=\varphi_{u}(t)+\varphi_{v}(t)
$$

where

$$
\begin{gathered}
\varphi_{u}(t)=\left[-\sigma^{n-1} y_{u}(t), \cdots,-y_{u}(t), \sigma^{n-1} u(t), \cdots, u(t)\right]^{T} \\
\varphi_{v}(t)=\left[-\sigma^{n-1} y_{v}(t), \cdots,-y_{v}(t), 0, \cdots, 0\right]^{T}
\end{gathered}
$$

Then

$$
R_{N}=R_{N}^{u}+R_{N}^{v}
$$

with obvious notation, and for (15) and (16) we have that

$$
R_{N} \geq \delta_{1} I
$$

for small enough $\delta$. Now, with

$$
\tilde{\theta}_{N}=\hat{\theta}_{N}-\theta_{0}
$$

we have

$$
\tilde{\theta}_{N}=R_{N}^{-1} \frac{1}{N} \sum_{t=1}^{N} \varphi(t) v(t)
$$




$$
|| \tilde{\theta}_{N}|| \leq \frac{1}{\delta_{1}} \cdot \delta \cdot \max \{|\varphi(t)|\} \leq C \cdot \delta
$$

We conclude that the least squares algorithm is robustly convergent for ARX-models provided $A(\sigma)$ has no roots on the unit circle (no roots on the imaginary axis), $A(\sigma)$ and $B(\sigma)$ have no common roots and $u(t)$ is p.e. of order $2 n$. If there are less parameters than $n$ in $B(\sigma)$ the p.e. condition on $u(t)$ can be weakened. Suppose that $B(q)=b_{1} q^{n-1}+\ldots+b_{m} q^{n-m}\left(B(p)=b_{1} p^{m}+\ldots+b_{m}\right)$ then it can be shown, analogously, that $u(t)$ has to be p.e. of order $n+m$. The continuous case and the discrete case have to be treated separately here.

\section{Problem statement}

In section 1 some definitions for discrete time systems have been stated. The definitions will now be carried over to the continuous time case. Let $\mathcal{M}$ be a model structure (continuous or discrete time) which is a smoothly parametrized set of models

$$
\mathcal{M}=\left\{\mathcal{M}(\theta) \mid \theta \in \mathcal{D}_{\mathcal{M}}\right\}
$$

Let a system $\rho=\mathcal{M}\left(\theta_{0}\right)$ generate exact input-output data

$$
Z_{0}=\left\{y_{0}(t), u(t) ; t=1, \ldots, N \text { or } t \in[a, b]\right\}
$$

where $t=1, \ldots, N$ for the discrete time case and $t \in$ $[a, b]$ for the continuous time case. Let the disturbance corrupted data be written

$$
Z=\left\{y_{v}(t), u(t) ; t=1, \ldots, N \text { or } t \in[a, b]\right\}
$$

Here $y_{v}(t)$ depends on the disturbance $v(t)$ with $|v(t)| \leq$ $\delta \forall t, \quad 0 \leq \delta<\infty$ in the discrete case and in the continuous case $v(t)$ also has to be band limited $\left|v^{(i)}(t)\right|<$ $c_{i} \delta \quad 0 \leq c_{i}<\infty$.

Definition 3.1 A model structure $\mathcal{M}$ is said to be globally identifiable at $\theta_{*}$ with respect to $\mathcal{D}_{\mathcal{M}}$ if there exists an input signal $u_{*}$ such that the output signal $\bar{y}\left(\theta_{*}, u_{*}\right) \neq \emptyset$ and

$$
\bar{y}\left(\theta_{*}, u_{*}\right) \equiv \bar{y}\left(\theta, u_{*}\right) \quad \theta \in \mathcal{D}_{\mathcal{M}} \Rightarrow \theta_{*}=\theta
$$

Definition 3.2 A model structure $\mathcal{M}$ is robustly globally identifiable if there exists an identification algorithm $\mathcal{J}$ and a $Z$ such that

$$
\hat{\theta}=\mathcal{J}(Z)
$$

and

$$
\lim _{\delta \rightarrow 0}\left|\hat{\theta}-\theta_{0}\right|=0
$$

The model structure considered here are all those systems that can be described in the following form

$$
\begin{aligned}
& \dot{x}=f(x, u, \theta) \\
& y=h(x, u, \theta)
\end{aligned}
$$

with the states $x \in R^{n}$, output $y \in R^{m}$, input $u \in R^{k}$ and parameters $\theta \in R^{d}$. The functions $f$ and $h$ are assumed to be polynomial in the variables $x, u$ and $\theta$.

The disturbed system is considered to be

$$
\begin{aligned}
& \dot{z}=f_{v}(z, u, v, \theta) \\
& y_{v}=h_{v}(z, u, v, \theta)
\end{aligned}
$$

with $z \in R^{n}, y_{v} \in R^{m}, u \in R^{k}$ and $\theta \in R^{d}$. The disturbance $v \in R^{l}$ is bounded above and band limited, i.e. $\left|v_{i}^{(j)}\right|<c_{j} \delta \quad 0 \leq c_{j}, \delta<\infty \quad j=0,1, \ldots, n+d+1 \quad i=$ $1, \ldots, l$. It is assumed that the signals $u, v \in C^{n+d+1}[a, b]$, where $C^{n}[a, b]$ denotes the space of $n$ times continuously differentiable functions with $t \in[a, b]$. The functions $f_{v}$ and $h_{v}$ are polynomials in the variables $z, u, v, \theta$ such that $f_{v}(z, u, 0, \theta)=f(z, u, \theta)$ and $h_{v}(z, u, 0, \theta)=$ $h(z, u, \theta)$.

Given initial states $x(a)$ of the system (23) there exists a solution to (23) in some interval $t \in[a, b]$ (Birkhoff and Rota, 1969), (Arnold, 1987).

Theorem 3.1 If a solution exists to the system (23) with $t \in[a, b]$ then the solution for the system (24) also exists with $t \in[a, b]$ if $\delta$ is sufficiently small

Proof. The proof is done by using similar methods as for continuation of solutions in (Birkhoff and Rota, 1969)

The parameter space $\mathcal{D}_{\mathcal{M}}$ for this model structure is here assumed to be equal to $R^{d}$. However it may be a smaller set if the physics is taken into account. Finally, a differential polynomial $F(u, \dot{u}, \ldots, y, \dot{y}, \ldots)$ will here be denoted $F(u, y ; p)$ where $p=\frac{d}{d t}$.

\section{Global identifiability implies robust global identifiability}

It is shown in (Glad and Ljung, 1990a), (Glad and Ljung, $1990 \mathrm{~b}$ ) that a system (23) is globally identifiable if and only if there can be found differential equations in the form

$$
\Phi_{i}(u, y ; p)+\theta_{i} \Psi_{i}(u, y ; p)=0
$$

where $i=1, \ldots, d$ by differentiating, adding, scaling and multiplying the equations in (23). An algorithm for finding these polynomials is given in (Glad and Ljung, 1990a), (Glad and Ljung, 1990b). It can be shown by using the result in (Glad, 1988) that there are no higher derivatives than $n+d$ in the polynomials $\Phi_{i}(u, y ; p)$ and 
$\Psi_{i}(u, y ; p)$. Replacing $y$ with $y_{v}$ of the disturbed system (24) yields

$$
\Phi_{i}\left(u, y_{v} ; p\right)+\theta_{i} \Psi_{i}\left(u, y_{v} ; p\right)+\Upsilon_{i}\left(u, y_{v}, v, z, \theta ; p\right)=0
$$

where $\Upsilon_{i}\left(u, y_{v}, v, z, \theta ; p\right)$ is a polynomial in the parameter $\theta$ and the variables $u, y_{v}, v$ and their derivatives. The expression for $\Upsilon_{i}(\cdot)$ is given in the proof of theorem 4.1 below.

The identification algorithm can now be defined.

Definition 4.1 Let the identification algorithm $\mathcal{J}$ be

$$
\hat{\theta}_{i}=-\frac{\int_{a}^{b} \Phi_{i}\left(u, y_{v} ; p\right) \Psi_{i}\left(u, y_{v} ; p\right) d t}{\int_{a}^{b} \Psi_{i}^{2}\left(u, y_{v} ; p\right) d t}
$$

Here it is assumed that $\int_{a}^{b} \Psi_{i}^{2} d t \neq 0$, i.e., the signal $u$ is p.e. (Glad and Ljung, 1990a), (Glad and Ljung, $1990 \mathrm{~b})$. Using equation (25) in (27) gives that $\hat{\theta}=\theta$ in the undisturbed case (23).

Theorem 4.1 Assume the model (23) to be globally identifiable, as in (Glad and Ljung, 1990a), (Glad and Ljung, 1990b). Let the initial condition $x(a)$ and the control $u$ on $[a, b]$ be chosen such that (23) has a solution on $[a, b]$ and that the excitation conditions giving global identifiability, (25), (see (Glad and Ljung, 1990a), (Glad and Ljung, 1990b)) are satisfied. Then (22) is satisfied, i.e. the model is robustly globally identifiable.

\section{Lemma 4.1}

$$
\Phi_{i}\left(u, y_{v} ; p\right)+\theta_{i} \Psi_{i}\left(u, y_{v} ; p\right)+\Upsilon_{i}\left(u, y_{v}, v, z, \theta ; p\right)=0
$$

where $\Upsilon_{i}(\cdot)$ satisfies

$$
\left|\Upsilon_{i}\left(u, y_{v}, v, z, \theta ; p\right)\right| \leq C \cdot \delta
$$

for $\delta$ sufficiently small

Proof: Since the right hand sides of equation (23) and (24) are polynomials in the variables $u, x, \theta$ respectively $u, v, z, \theta$ and $u, v \in C^{n+d+1}$ the solutions $x(t)$ and $z(t)$ are $n+d$ times continuously differentiable functions (Arnold, 1987). It also follows that $z(t)$ and its $n+d$ derivatives converges uniformly to $x(t)$ and the corresponding derivatives (Birkhoff and Rota, 1969). The output signals $y_{v}, y$ and their derivatives up to order $n+d$ can now be obtained from (23) and (24). Furthermore $y_{v}^{(j)}(t)$ converges uniformly to $y^{(j)}(t) \quad j=0, \ldots, n+d$. This follows from the fact that $f_{v}, h_{v}$ are polynomial in the variables $z, u, v, \theta$ and converges to $f, h,\left|v_{i}^{(j)}\right|<c_{j} \delta$ and $u, v \in C^{n+d+1}$. Polynomials in $y_{v}(t)$ and its derivatives will also converge uniformly to the corresponding polynomials in $y(t)$ and its derivatives.
Introduce the notion

$$
\left[\begin{array}{l}
A_{1}(u, \theta, x ; p) \\
\vdots \\
A_{n}(u, \theta, x ; p) \\
A_{n+1}(u, y, \theta, x) \\
\vdots \\
A_{n+m}(u, y, \theta, x)
\end{array}\right]=\left[\begin{array}{l}
\dot{x}_{1}-f_{1}(x, u, \theta) \\
\vdots \\
\dot{x}_{n}-f_{n}(x, u, \theta) \\
y_{1}-h_{1}(x, u, \theta) \\
\vdots \\
y_{m}-h_{m}(x, u, \theta)
\end{array}\right] .
$$

for the system (23). The polynomials (25) can then be expressed as

$$
\begin{gathered}
\Phi_{i}(u, y ; p)+\theta_{i} \Psi_{i}(u, y ; p)= \\
\sum_{i, j} Q_{i j}(u, y, \theta, x ; p) A_{i}^{(j)}(u, y, \theta, x)
\end{gathered}
$$

where the sum is finite and $Q_{i j}$ are some polynomials in the parameter $\theta$ and the variables $u, y, x$ and their derivatives. Similarly introduce the notion $A_{v, i}(u, y, \theta, z ; p)$ for the system (24). Changing $y$ and $x$ to $y_{v}$ and $z$ in the expression (29) gives

$$
\begin{gathered}
\Phi_{i}\left(u, y_{v} ; p\right)+\theta_{i} \Psi_{i}\left(u, y_{v} ; p\right)= \\
\sum_{i, j} Q_{i j}\left(u, y_{v}, \theta, z ; p\right) A_{i}^{(j)}\left(u, y_{v}, \theta, z\right)= \\
\underbrace{\sum_{i, j} Q_{i j}\left(u, y_{v}, \theta, z ; p\right) A_{v, i}^{(j)}\left(u, y_{v}, \theta, z, v\right)+}_{=0}
\end{gathered}
$$

$\underbrace{\sum_{i, j} Q_{i j}\left(u, y_{v}, \theta, z ; p\right)\left(A_{i}^{(j)}\left(u, y_{v}, \theta, z\right)-A_{v, i}^{(j)}\left(u, y_{v}, \theta, z, v\right)\right)}_{-\Upsilon_{i}\left(u, y_{v}, v, z, \theta ; p\right)}$

which can be compared with (26). Now

$$
\begin{gathered}
\left|\Upsilon_{i}\left(u, y_{v}, v, z, \theta ; p\right)\right| \leq \\
\sum_{i, j}\left|Q_{i j}\left(u, y_{v}, \theta, z ; p\right)\right| \cdot \\
\left(c_{0}^{i j}|| v\left\|+c_{1}^{i j}\right\| \dot{v}\left\|+\ldots+c_{n+d}^{i j}|| v^{(n+d)}\right\|\right) \leq C \cdot \delta
\end{gathered}
$$

for some constants $0 \leq c_{k}^{i j}<\infty$

\section{Proof of Theorem 4.1}

If the system (23) is globally identifiable then there are expressions of the form (26). The identification algorithm $\mathcal{J}$ gives

$$
\begin{gathered}
\hat{\theta}_{i}=-\frac{\int_{a}^{b} \Phi_{i}\left(u, y_{v} ; p\right) \Psi_{i}\left(u, y_{v} ; p\right) d t}{\int_{a}^{b} \Psi_{i}^{2}\left(u, y_{v} ; p\right) d t}= \\
\theta_{i}+\frac{\int_{a}^{b} \Upsilon_{i}\left(u, y_{v}, v, z, \theta ; p\right) \Psi_{i}\left(u, y_{v} ; p\right) d t}{\int_{a}^{b} \Psi_{i}^{2}\left(u, y_{v} ; p\right) d t}
\end{gathered}
$$


We will show that

$$
\frac{\int_{a}^{b} \Upsilon_{i}\left(u, y_{v}, v, z, \theta ; p\right) \Psi_{i}\left(u, y_{v} ; p\right) d t}{\int_{a}^{b} \Psi_{i}^{2}\left(u, y_{v} ; p\right) d t} \rightarrow 0, \quad \delta \rightarrow 0
$$

It follows from Lemma 4.1 that

$$
\Upsilon_{i}\left(u, y_{v}, v, z, \theta ; p\right) \rightarrow 0 \quad \delta \rightarrow 0
$$

uniformly. Using the result in the first part of the proof of Lemma 4.1 it can be shown that

$$
\begin{gathered}
\Psi_{i}\left(u, y_{v} ; p\right) \rightarrow \Psi_{i}(u, y ; p) \quad \delta \rightarrow 0 \\
\Phi_{i}\left(u, y_{v} ; p\right) \rightarrow \Phi_{i}(u, y ; p) \quad \delta \rightarrow 0
\end{gathered}
$$

uniformly. Uniform convergence also implies that

$$
\begin{gathered}
\int_{a}^{b} \Psi_{i}^{2}\left(u, y_{v} ; p\right) \rightarrow \int_{a}^{b} \Psi_{i}^{2}(u, y ; p) \delta \rightarrow 0 \\
\int_{a}^{b} \Phi_{i}\left(u, y_{v} ; p\right) \Upsilon_{i}\left(u, y_{v}, v, z, \theta ; p\right) \rightarrow 0 \quad \delta \rightarrow 0
\end{gathered}
$$

Since $\Psi_{i}^{2}\left(u, y_{v} ; p\right)$ is continuous and converges uniformly to $\Psi_{i}^{2}(u, y ; p)$ and $\int_{a}^{b} \Psi_{i}^{2}(u, y ; p) \neq 0$ then $\int_{a}^{b} \Psi_{i}^{2}\left(u, y_{v} ; p\right) \neq 0$ if $\delta$ sufficiently small.

Consequently (32) holds so that $\hat{\theta} \rightarrow \theta_{0}, \quad \delta \rightarrow 0$

\section{Conclusions}

We have studied a class of systems which can be written in state space form $\dot{x}=f(u, x, \theta), \quad y=h(u, x, \theta)$ where $f$ and $h$ are polynomials in $u, x$ and $\theta$. We have shown that if such a state space system is globally identifiable its disturbed counterpart is robustly globally identifiable. We also remark that similar techniques can be used to establish the result for nonlinear discrete time state space systems.

\section{Acknowledgement}

This work has been supported by the Swedish Research Council for Engineering Sciences (TFR).

\section{References}

Akçay, H. (1992). Robust linear system identification in $H_{\infty}$. PhD thesis, University of Michigan.

Arnold, V. I. (1987). Ordinary Differential Equations. The MIT Press, Cambridge, Massachusettes.

Åström, K. J. and Wittenmark, B. (1989). Adaptive Control. Addison Wesley Publishing Company.

Birkhoff, G. and Rota, G.-C. (1969). Ordinary Differential Equations. Blaisdell Publishing Company, Waltham, Massachusetts.
Chen, J., Nett, C. N., and Fan, M. K. H. (1992). Optimal non-parametric system identification from arbitrary corrupt finite time series: A control-oriented approach. In Proc. $A C C$.

Glad, S. (1988). Nonlinear state space and input output descriptions using differential polynomials. Technical report, Technical Report LiTH-ISY-I-0964, Department of Electrical Engineering, Linköping University, S-581 83 Linköping, Sweden.

Glad, S. and Ljung, L. (1990a). Model structure identifiability and persistence of excitation. In Proc. of the 29th IEEE Conference on Decision and Control, Honolulu, Hawaii, pages 5.7-5.12.

Glad, S. and Ljung, L. (1990b). Parametrization of nonlinear model structures as linear regressions. In Preprints of 11th IFAC World Congress, Tallin, Estonia, pages $67-71$.

Gu, G. and Khargonekar, P. (1992). A class of algorithms for identification in $H_{\infty}$. Automatica, 28:299-312.

Helmicki, A. J., Jacobson, C. A., and Nett, C. N. (1991). "Control Oriented System Identification: A WorstCase/Deterministic Approach in $H_{\infty}$ ". IEEE Transactions on Automatic Control, AC-36:1163-1176.

Helmicki, A. J., Jacobson, C. A., and Nett, C. N. (1992). "Worst-Case/Deterministic Identification in $H_{\infty}$ :The Continuous Time Case". IEEE Transactions on Automatic Control, AC-37:604-609.

Ljung, L. (1987). System Identification: Theory for the User. Prentice-Hall, Englewood Cliffs, NJ.

Ljung, L. and Wahlberg, B. (1992). Asymptotic properties of the least-squares method for estimating transfer functions and disturbance spectra. Adv. Appl. Prob., $24: 412-440$.

Ljung, L. and Yuan, Z. D. (1985). "Asymptotic properties of black-box identification of transfer functions". IEEE Trans. Automat. Contr., AC-30:514-530.

Mäkilä, P. M. (1991). "Robust Identification and Galois Sequences". International Journal of Control, 54:1189-1200. 